\title{
Bacteremia in Pediatric Gastroenterology Patients with Central Venous Catheters Presenting to the Emergency Department
}

\author{
Courtney E Brennan, $M D^{1^{*}}$, Choo Phei Wee, $M S^{2}$, Jared Schiff, $M D^{1}$ and Alan L Nager, MD, MHA \\ ${ }^{1}$ Division of Emergency and Transport Medicine, Department of Pediatrics, Children's Hospital Los Angeles, \\ Keck School of Medicine of the University of Southern California, USA \\ ${ }^{2}$ Biostatistician II, Biostatistics Core, Clinical Research Support Office, Children's Hospital Los Angeles, USA
}

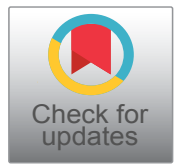

*Corresponding author: Courtney E Brennan, MD, Division of Emergency and Transport Medicine, Department of Pediatrics, Children's Hospital Los Angeles, Keck School of Medicine/University of Southern California, 4650 Sunset Blvd, MS \# 113, Los Angeles, CA 90027, USA, Tel: 323-361-6522, Fax: 323-361-3891

\begin{abstract}
Objectives: The purpose of this study is to determine if there are clinical and laboratory results that can predict bacteremiain pediatric gastroenterology $(\mathrm{GI})$ patients with central venous catheters (CVCs) presenting to the Emergency Department (ED) with fever.

Methods: Medical records were retrospectively reviewed for gastroenterology patients presenting to a single, Pediatric Emergency Department with fever and a CVC from April 1, 2014 to December 31, 2016. The primary outcome measure is a positive blood culture. Additional variables studied include age, sex, history of prematurity, primary GI diagnosis, type of CVC and when it was inserted, the presence or absence of an enteral feeding tube, the use of total parenteral nutrition (TPN), weight, ED presenting and discharge temperature, heart rate, respiratory rate, blood pressure and oxygen saturation, the amount of intravenous fluid given, the presence of upper respiratory infection (URI) symptoms such as cough, congestion and rhinorrhea, laboratory values including total white blood cell count, absolute neutrophil count (ANC), platelet count, C-reactive protein (CRP), urinalysis results, and whether the patient was discharged home, admitted to the general ward or admitted to the intensive care unit (ICU). Data were analyzed using descriptive statistics.
\end{abstract}

Results: One hundred thirty-one ED visits were sampled and 110 encounters were included in the study. Seventy-five patients were bacteremic $(68.2 \%)$, and the most common pathogen isolated from blood cultures was coagulase-negative Staphylococci species. Of the variables studied, only total platelet count showed a significant association with bacteremia, with a lower mean platelet count in the bacteremia group.
Conclusion: The incidence of bacteremia in febrile GI patients with CVCs is high. Lower total platelet count is associated with bacteremia in this group. However, platelet count alone should not be used as a predictor of bacteremia. The current practice of admitting these patients to the hospital on empiric antibiotics while awaiting blood culture results and susceptibilities remains prudent.

\section{Introduction}

The presence of a central venous catheter (CVC) is an independent risk factor for central line associated blood stream infection (CLABSI) in all pediatric patients. Previous studies have shown the incidence of CLABSI in febrile pediatric gastroenterology (GI) patients, and in particular pediatric patients with intestinal failure, to be as high as $47-69 \%$ [1-3]. This is much higher than in other pediatric patient populations with CVCs, such as the pediatric oncology population, in which the incidence of bacteremia is only as high as $39 \%$ in both neutropenic and non-neutropenic patients [4].

Pediatric GI patients require CVCs for various reasons, however, most commonly this is due to the need for parenteral nutrition in the setting of intestinal failure. Intestinal failure (IF) is defined as the"reduction of gut function below the minimum necessary for the absorption of macronutrients and/or water and electrolytes, such that intravenous supplementation is required to maintain health and/or growth [5]". In the pediatric population, this is often due to short bowel syndrome,

Citation: Brennan CE, Wee CP, Schiff J, Nager AL (2021) Bacteremia in Pediatric Gastroenterology Patients with Central Venous Catheters Presenting to the Emergency Department. Int J Pediatr Res 7:074. doi.org/10.23937/2469-5769/1510074

Accepted: April 19, 2021: Published: April 21, 2021

Copyright: (C) 2021 Brennan CE, et al. This is an open-access article distributed under the terms of the Creative Commons Attribution License, which permits unrestricted use, distribution, and reproduction in any medium, provided the original author and source are credited. 
but there are many other causes. Parenteral nutrition made IF a survivable disease when it was introduced over 50 years ago, but this disease still carries a significant burden, with mortality rates from 25 to $37.5 \%$ [68]. CLABSI is a significant cause of morbidity and mortality in IF patients $[9,10]$. If patients account for up to $20 \%$ of all bloodstream infection hospital admissions, the majority of which have CVCs [11]. The reason for such high rates of bacteremia in IF patients goes beyond skin flora being introduced into the CVC, and is multifactorial [12]. These factors include, bacterial translocation from the intestine, increased mucosal inflammation, impaired or dysregulated immune function, increased gut permeability, an altered microbiome, contamination of the TPN, or direct contamination of the line from stool [13-16].

As a result of these unique risk factors, there is an increased concern for blood stream infectionsinpediatric GI patients with CVCs whopresent to the Emergency Department (ED) when febrile. Due to the high incidence of bacteremia and concern for resultant morbidity and mortality, these patients are frequentlyadmitted to the hospital and started on empiric antibiotics while awaiting culture results and sensitivity testing.

As there are few previous studies looking at predictors of bacteremia, we sought to determine if there are clinical and laboratory results associated with bacteremia in pediatric GI patients with a CVC who present to the ED with fever.

\section{Methods}

This is a retrospective cohort study conducted at a large, urban, tertiary care children's hospital. The pediatric ED at this institution sees $>90,000$ patient visits each year, and the hospital is a large referral center for pediatric GI patients. The Institutional Review Board at the study hospital approved this study.

An electronic query of patient medical records was performed to identify ED visits between April 1, 2014 and December 31, 2016 with a documented chief complaint of fever and an associated International Classification of Diseases, Ninth or Tenth Edition primary gastroenterology diagnosis.Patients were included if they were 21 years of age, had an indwelling CVC present, had a temperature of $\geq 38$ Celsius documented at the time of the ED visit or reported by the caretaker prior to arrival to the ED, and had a blood culture obtainedfrom the CVC upon ED presentation. Exclusion criteria included, any patients transferred to the ED from an outside hospital, patients who had any antibiotics in the 24 hours prior to the ED visit, patients discharged from any hospital in the 48 hours preceding the ED visit, or patients receiving total parenteral nutrition (TPN) for any reason other than a primary GI diagnosis. Some patients had more than one encounter, and each eligible encounter was included in the analysis.
All data were extracted by 2 study personnel, CEB and JS. An electronic data collection form was created using Research Electronic Data Capture (REDcap) [17] hosted by the study institutionand all data were entered directly into this data form. Inter-rater reliability was calculated with the first 30 patient records; initial reliability statistic was moderate (Cohen's Kappa $=0.78$ ) between the 2 retrospective reviewers. Following inter-rater reliability calculations, the 2 reviewers met and discussed discrepancies and came to a consensus on subsequent reviews.

The primary outcome for the analysis was bacteremia which was defined by a microbiology laboratory-confirmed bacterial or fungal species growth from the blood sample taken from the CVC upon presentation to the ED. These were considered to be real and not contaminants if the patient received a treatment course of antibiotics or antifungals. Bacteremia was chosen as the outcome measure instead of the commonly used definition, CLABSI, as we determined that this would capture all patients receiving a treatment course of antibiotics or antifungals at our institution due to a positive blood culture and would not exclude patients for which another source of infection was also possible. Peripheral blood cultures as well as anaerobic were not routinely drawn in the ED during the study time period, and therefore are not included in the analysis. Additionally, the catheter tip was not routinely sent for culture from the ED, so was also not included in the analysis. The following independent variables were determined, a priori, and extracted from the chart for each encounter: age, sex, history of prematurity, primary GI diagnosis, type of CVC and when it was inserted, the presence or absence of an enteral feeding tube, the use of TPN, weight, ED presenting and discharge temperature, heart rate, respiratory rate, blood pressure and oxygen saturation,the amount of intravenous fluid given, the presence of upper respiratory infection (URI) symptoms such as cough, congestion and rhinorrhea, laboratory values including total white blood cell count, absolute neutrophil count (ANC), platelet count, CRP, urinalysis results, and whether the patient was discharged home, admitted to the general ward or admitted to the intensive care unit (ICU).

A minimum sample size of 98 independent cases was proposed. This achieved a desired study power of $80 \%$ to detect an incidence of positive blood culture of 50$65 \%$. These calculations were based upon the incidence of positive blood cultures from previously published literature [1-3]. Continuous variables were summarized as a mean with standard deviation and median with interquartile range $\left(25^{\text {th }}\right.$ percentile $-75^{\text {th }}$ percentile). Frequency and percentages were used to summarize categorical variables. A two-sample t-test was used to compare the mean difference for continuous variables that are normally distributed between negative and positive blood cultures. Quantile regression analysis, 
a non-parametric approach, was used to compare the median difference for continuous variables that are not normally distributed between the two comparison groups. For categorical variables, chi-square test was used when the expected cell count was more than 5 , and Fishers exact test was used when the expected cell count was less than 5 . To assess the association in age in 6 months intervals with bacteremia, logistic regression analysis was used. Statistical significance was set at 0.05 with two-sided analysis throughout. All statistical analyses were performed using Stata 15.1 Intercooled (StataCorp College Station, Texas).

\section{Results}

One hundred thirty-one ED visits were sampled. A total of 110 febrile encounters were included in the study. Of the 21 excluded patients, 13 did not have a central venous catheter present at the time of the encounter, 5 did not have fever or a history of fever, 2 did not have primary GI diagnoses, and 1 patient presented after leaving against medical advice from another institution where he had been receiving antibiotics. Several patients had more than one encounter.

Table 1 describes thecharacteristics of the patients

Table 1: The characteristics of the patients included in the analysis.

\begin{tabular}{|c|c|c|c|}
\hline & $\begin{array}{l}\text { Bacteremia negative } \\
(\mathrm{N}=35)\end{array}$ & $\begin{array}{l}\text { Bacteremia } \\
\text { Positive } \\
(\mathrm{N}=75)\end{array}$ & $\begin{array}{l}\text { Measure of association/ } \\
\text { differences } \\
\text { P-value }\end{array}$ \\
\hline Gender & & & $0.462^{* *}$ \\
\hline Female & $11(31.43 \%)$ & $29(38.67 \%)$ & \\
\hline Male & $24(68.57 \%)$ & $46(61.33 \%)$ & \\
\hline Age (in years) & $3.70( \pm 2.43)$ & $4.24( \pm 3.09)$ & $0.3561^{* *}$ \\
\hline Weight (in kilograms) & $15.89( \pm 6.36)$ & $16.80( \pm 7.60)$ & $0.5415^{* *}$ \\
\hline Short bowel syndrome & & & $0.094^{*}$ \\
\hline No & $3(8.57 \%)$ & $1(1.33 \%)$ & \\
\hline Yes & $32(91.43 \%)$ & $74(98.67 \%)$ & \\
\hline $\begin{array}{l}\text { Intestinal obstruction/pseudo } \\
\text { obstruction }\end{array}$ & & & $0.537^{*}$ \\
\hline No & $34(97.14 \%)$ & $74(98.67 \%)$ & \\
\hline Yes & $1(2.86 \%)$ & $1(1.33 \%)$ & \\
\hline Other GI diagnosis & & & $0.237^{*}$ \\
\hline No & $33(94.29 \%)$ & $74(98.67 \%)$ & \\
\hline Yes & $2(5.71 \%)$ & $1(1.33 \%)$ & \\
\hline History of prematurity & & & $0.189^{\wedge}$ \\
\hline No & $11(31.43 \%)$ & $15(20 \%)$ & \\
\hline Yes & $24(68.57 \%)$ & $60(80 \%)$ & \\
\hline Type of central line & & & $0.380^{*}$ \\
\hline PICC & $3(8.57 \%)$ & $3(4 \%)$ & \\
\hline Broviac $^{\circledR}$ & $32(91.43 \%)$ & $72(96 \%)$ & \\
\hline Number of CVC lumens & & & NA \\
\hline 1 & $35(100 \%)$ & $74(98.67 \%)$ & \\
\hline 2 & $0(0 \%)$ & $1(1.33 \%)$ & \\
\hline $\begin{array}{l}\text { TPN administered through central } \\
\text { line }\end{array}$ & & & $0.537^{*}$ \\
\hline No & $1(2.86 \%)$ & $1(1.33 \%)$ & \\
\hline Yes & $34(97.14 \%)$ & $74(98.57 \%)$ & \\
\hline Additional enteral nutrition & & & $0.999^{*}$ \\
\hline No & $1(2.86 \%)$ & $3(4.00 \%)$ & \\
\hline Yes & $34(97.14 \%)$ & $72(96 \%)$ & \\
\hline G-tube or GJ tube present? & & & $0.873^{\wedge}$ \\
\hline No & $7(20 \%)$ & $16(21.33 \%)$ & \\
\hline Yes & $28(80 \%)$ & $59(78.67 \%)$ & \\
\hline Any prior bacteremia & & & $0.999^{*}$ \\
\hline
\end{tabular}




\begin{tabular}{|c|c|c|c|}
\hline No & $2(5.71 \%)$ & $6(8 \%)$ & \\
\hline Yes & $33(94.29 \%)$ & $69(92 \%)$ & \\
\hline Fever measured prior to arrival & & & $0.267^{*}$ \\
\hline No & $1(2.86 \%)$ & $8(10.67 \%)$ & \\
\hline Yes & $34(97.14 \%)$ & $67(89.33 \%)$ & \\
\hline Height of fever at home & $101.45( \pm 0.93)$ & $101.86( \pm 1.06)$ & $0.0642^{\star *}$ \\
\hline $\begin{array}{l}\text { Presence of upper respiratory } \\
\text { symptoms }\end{array}$ & & & $0.665^{\wedge}$ \\
\hline No & $19(54.29 \%)$ & $44(58.67 \%)$ & \\
\hline Yes & $16(45.71 \%)$ & $31(41.33 \%)$ & \\
\hline Triage/Initial temperature $\left(C^{\circ}\right)$ & $37.96( \pm 0.98)$ & $38.17( \pm 1.04)$ & $0.3107^{* *}$ \\
\hline Triage/Initial heart rate & $147( \pm 25.26)$ & $152.28( \pm 25.87)$ & $0.3175^{\star *}$ \\
\hline Triage/Initial respiratory rate & $27.08( \pm 5.30)$ & $29.55( \pm 9.71)$ & $0.1634^{* *}$ \\
\hline $\begin{array}{l}\text { Triage/Initial systolic blood } \\
\text { pressure }\end{array}$ & $110.81( \pm 11.52)$ & $111.82( \pm 17.42)$ & $0.7684^{* *}$ \\
\hline $\begin{array}{l}\text { Triage/Initial diastolic blood } \\
\text { pressure }\end{array}$ & $68.48( \pm 11.60)$ & $66.39( \pm 11.77)$ & $0.4146 *$ \\
\hline Triage/Initial pulse oximetry & $98.8( \pm 1.35)$ & $98.34( \pm 2.94)$ & $0.3777^{* *}$ \\
\hline Total white blood cell count & $7.3(3.96-10.76)$ & $6.66(4.69-10.8)$ & $0.613 \sim$ \\
\hline Total absolute neutrophil count & $4.09(2.44-6.97)$ & $4.98(2.9-7.35)$ & $0.320 \sim$ \\
\hline CRP & $3.9(2-6.1)$ & $4.3(2.9-6.6)$ & \\
\hline Urine dip and/or micro obtained & & & $0.551^{\wedge}$ \\
\hline No & $17(48.57 \%)$ & $41(54.67 \%)$ & \\
\hline Yes & $18(51.43 \%)$ & $34(45.33 \%)$ & \\
\hline Urine leukocyte esterase & & & $0.155^{\wedge}$ \\
\hline Negative & $15(42.86 \%)$ & $28(37.33 \%)$ & \\
\hline Trace & $0(0 \%)$ & $3(4 \%)$ & \\
\hline Small & $0(0 \%)$ & $2(2.67 \%)$ & \\
\hline Moderate & - & - & \\
\hline Large & - & - & \\
\hline Unknown & $3(8.57 \%)$ & $1(1.33 \%)$ & \\
\hline Urine nitrite & & & $0.178^{*}$ \\
\hline Negative & $15(42.86 \%)$ & $32(42.67 \%)$ & \\
\hline Positive & $0(0 \%)$ & $1(1.33 \%)$ & \\
\hline Unknown & $3(8.57 \%)$ & $1(1.33 \%)$ & \\
\hline Urine microscopy count & & & $0.074^{*}$ \\
\hline $0-3$ & $5(14.29 \%)$ & $8(10.67 \%)$ & \\
\hline $3-5$ & $0(0 \%)$ & $2(2.67 \%)$ & \\
\hline $5-10$ & $3(8.57 \%)$ & $0(0 \%)$ & \\
\hline No micro obtained & $10(28.57 \%)$ & $24(32 \%)$ & \\
\hline $\begin{array}{l}\text { Total fluid bolus given in ED } \\
\text { (milliliters) }\end{array}$ & $340(0-624)$ & $475(80-800)$ & $0.312 \sim$ \\
\hline Total fluid bolus/weight (kg) & $20.08(0-40.12)$ & $29.20(18.74-57.06)$ & $0.137 \sim$ \\
\hline \multicolumn{4}{|l|}{$\begin{array}{l}\text { Total fluid bolus/weight (kg) } \\
\text { category }\end{array}$} \\
\hline $0-20$ & $17(48.57 \%)$ & $29(38.67 \%)$ & $0.068^{*}$ \\
\hline$>20-40$ & $9(25.71 \%)$ & $22(29.33 \%)$ & \\
\hline$>40-60$ & $7(20 \%)$ & $7(9.33 \%)$ & \\
\hline$>60$ & $2(5.71 \%)$ & $17(22.67 \%)$ & \\
\hline Disposition & & & $0.305^{\star}$ \\
\hline Admit to floor & $35(100 \%)$ & $71(94.67 \%)$ & \\
\hline
\end{tabular}




\begin{tabular}{|l|l|l|l|}
\hline \multicolumn{1}{|c|}{ Admit to NICU or PICU } & $0(0 \%)$ & $4(5.33 \%)$ & NA \\
\hline Reason for ICU admission & & & \\
\hline Vasoactive meds needed & $0(0 \%)$ & $3(4 \%)$ & $1(1.33 \%)$ \\
\hline Other & $0(0 \%)$ & $37.17( \pm 0.69)$ & $0.9585^{* *}$ \\
\hline Discharge temp (C) & $37.17( \pm 0.77)$ & $129.05( \pm 21.34)$ & $0.4901^{* *}$ \\
\hline Discharge heart rate & $126.09( \pm 20.02)$ & $27.75( \pm 8.04)$ & $0.2166^{* *}$ \\
\hline Discharge respiratory rate & $25.89( \pm 5.41)$ & $101.14( \pm 12.85)$ & $0.3978^{* *}$ \\
\hline $\begin{array}{l}\text { Discharge systolic blood } \\
\text { pressure }\end{array}$ & $103.20( \pm 9.36)$ & $61.86( \pm 13.59)$ & $0.0675^{* *}$ \\
\hline $\begin{array}{l}\text { Discharge diastolic blood } \\
\text { pressure }\end{array}$ & $66.83( \pm 11.97)$ & $98.30( \pm 5.53)$ & $0.4396^{* *}$ \\
\hline Discharge pulse oximetry & $99.06( \pm 1.20)$ & & $0.491^{\wedge}$ \\
\hline Urine culture obtained & & $48(64 \%)$ & \\
\hline No & $20(57.14 \%)$ & $27(36 \%)$ & $146(99-196)$ \\
\hline Yes & $15(42.86 \%)$ & & $0.043^{*}$ \\
\hline Platelets & $186(117-262)$ & & \\
\hline
\end{tabular}

$\wedge:$ Statistical difference is based on Chi-Square test.

*: The difference in mean between two groups is based on two sample t-test.

$\sim:$ The difference in median between two groups is based on quantile regression model.

*: Statistical differences are based on Fisher's exact test.

Table 2: List of pathogens detected from blood cultures.

\begin{tabular}{|l|l|}
\hline Central blood culture pathogens $\mathbf{N}=\mathbf{7 5})$ & $\mathbf{N}(\%$ of total positive cultures) \\
\hline Coagulase-negative Staphylococcus species & $29(38.7 \%)$ \\
\hline Staphylococcus aureus & $10(13.3 \%)$ \\
\hline Klebsiella species & $10(13.3 \%)$ \\
\hline Enterobacter species & $6(8 \%)$ \\
\hline Mixed pathogens & $6(8 \%)$ \\
\hline Enterococcus species & $3(4 \%)$ \\
\hline Serratia marcescens & $3(4 \%)$ \\
\hline Bacillus cereus & $2(2.7 \%)$ \\
\hline Escherichia coli & $2(2.7 \%)$ \\
\hline Candida species & $2(2.7 \%)$ \\
\hline Citrobacter freundii & $1(1.3 \%)$ \\
\hline Leuconostoc species & $1(1.3 \%)$ \\
\hline
\end{tabular}

included in the analysis. A blood culture was positive in $75 / 110(68.2 \%)$ encounters included in the study. The most common pathogens isolated from blood cultures were Coagulase negative staph species, followed by Klebsiella species and Staphylococcus aureus species. Candida species was detected in 2 blood cultures and there were 6 polymicrobial infections (Table 2). One hundred six patients had an underlying diagnosis of IF. This was most commonly due to short bowel syndrome (SBS) secondary to gastroschisis or necrotizing enterocolitis.

The only statistically significant association with the presence of bacteremia was the total platelet count $(p=$ $0.043)$, with lower median total platelet count $(143$, IQR 99-196) in the bacteremia group vs. the non-bacteremia group (186, IQR 117-262).

All patient encounters resulted in admission to the hospital. Four patients (3.6\%), all of whom were bacteremic, resulted in admission to the intensive care unit (ICU) from the ED for further management. No patients in the non-bacteremia group required ICU admission. Of the patients requiring ICU admission, 3 also required vasoactive medications i.e., Dopamine.

\section{Discussion}

In this study of children with primary Gl diagnoses and CVCs presenting to the ED with fever, the incidence of bacteremia was high, occurring in $68.2 \%$ of encounters. This is similar to the incidence reported in previous literature.

With CLABSIs contributing to such a high morbidity and mortality rate, it is helpful to determine which $\mathrm{GI}$ patients with a fever and CVC are more likely to be bacteremic. Few previous studies have examined risk fac- 
tors for CLABSIs in pediatric patients with IF, but there is evidence that elevated absolute neutrophil count, low total white blood cell count, thrombocytopenia, elevated C-reactive protein (CRP), age $<1$ year, and temperature $\geq 39^{\circ} \mathrm{C}$ were associated with higher rates of bacteremia $[1,2,9,18,19]$.

Bacteremia was associated with a lower total platelet count in the current study. This is similar to the findings in a recent study by Eisenberg et al which also showed that thrombocytopenia was associated with bacteremia in pediatric IF patients with a CVC presenting to the ED with fever [2]. Thrombocytopenia is common in patients in the setting of bacteremia, sepsis, and septic shock. The mechanisms for this are complex and may be due to altered thrombopoiesis due to inflammation and endothelial dysfunction and coagulopathy [20]. Thrombocytopenia is also common in patients with intestinal failure associated liver disease. This is likely due to the decreased production of thrombopoietin in the diseased liver and to portal hypertension causing increased splenic consumption [21].

In the study by Eisenberg, et al. they found that in addition to low platelets, height of fever and low total white blood cell counts were also associated with CLABSI. Their findings were similar to previous studies showing association between these variables and serious bacterial infection. However, we did not find the same associations in the current study. This may be due to several factors including patient demographics, study site location, and the higher incidence of bacteremia caused by gram-positive organisms in our study. Although these risk factors for bacteremia were identified, the study still found the incidence of bacteremia to be $25.4 \%$ even when all the identified risk factors were absent. Thus, the study authors concluded that these patients could not be risk-stratified into high and lowrisk of bacteremia and all IF patients should be treated with empiric broad-spectrum antibiotics. In our study population, that is almost entirely comprised of patients with an underlying diagnosis of IF, it is difficult to determine if the association of low platelets with the bacteremia group is truly due to the presence of the bacterial infection and not confounded by the presence of possible intestinal failure associated liver disease as the number of patients with IF who also had intestinal failure associated liver disease was not specifically studied. We therefore caution the use of lower total platelet count as a reliable predictor of bacteremia in this patient population.

Forty percent of bacteremia cases in the current study were due to Coagulase negative staph species. In several studies, gram negative organisms accounted for the majority of bacteremia cases. This high incidence of Coagulase negative Staph may be due to the fact that for all cases in which a Coagulase negative Staph species grew from the initial blood culture, these patients were treated with a full course of antibiotics. This was the case even in some instances when the culture result was positive from only one of the CVC lumens. Some of these CVCs may have been colonized with these bacteria, and thus a peripheral culture or repeat culture may not have yielded a positive result. Therefore, the incidence of true bacteremia may be elevated in the current study.

Only 4 (3.4\%) of patients in the current study required admission to an intensive care unit. Previous studies have cited a higher percentage of patients requiring intensive care management (9-11.5\%) [1-3]. Although there was not a statistically significant association between ICU admission and bacteremia in this study, all four patients admitted to an ICU in this study had blood cultures growing an organism meeting the study definition of bacteremia. Each of these patients required extensive fluid resuscitation of more than 60 $\mathrm{ml} / \mathrm{kg}$ in the ED and three of the patients required vasoactive medication initiation prior to ICU admission. Additional similarities between these patients included a primary diagnosis of IF, age less than two years, and tachycardia upon arrival to the ED.

Additionally, though not statistically significant, $22.7 \%$ percent of patients with bacteremia required extensive fluid resuscitation of $60 \mathrm{ml} / \mathrm{kg}$ or more vs. only $5.7 \%$ in the non-bacteremia group. Intravenous fluid administration is crucial in the management of sepsis and septic shock. Since fluid was administered at the discretion of the treating physician, there were likely vital sign, physical exam, or laboratory abnormalities in the bacteremia group that triggered the treating physicians to deem this group more ill and in need of more aggressive fluid management. It is possible that this extensive fluid resuscitation may have stabilized some patients, and therefore, obviated the need for ICU admission.

\section{Limitations}

There are several limitations to this study. Given the retrospective nature of the study, it is possible that we may have missed some eligible encounters of pediatric GI patients presenting to the ED. Some variables were missing from some of the encounters and thus were not included in the analysis. Although there was adequate agreement between the study personnel extracting data, there was not complete agreement between the parties and therefore there may have been some discrepancies in the data that was extracted.

Laboratory studies were ordered at the discretion of the treating physician and not as part of a protocol. Therefore, not all of the studied laboratory data were obtained on each patient making the association with laboratory values and bacteremia difficult to interpret. During the time of the study, peripheral blood cultures and anaerobic blood cultures were not routinely ordered at our institution and therefore not included in 
the study. This may have resulted in an over or underestimation of the number of true cases of bacteremia. Similarly, the amount of normal saline given to each patient was also at the discretion of the treating physician which allowed for some interpretation. The current study also did not look at age adjusted vital signs. While we did not find any statistically significant association between vital signs and bacteremia, it is possible that if vital signs were adjusted for age there may have been an association.

The outcome measure we chose for this study, bacteremia, included all patients with a positive blood culture who received a treatment course of antibiotics. This does not specifically exclude patients with the possibility of another source of infection. We chose to define the outcome this way in order to reflect the clinical practice at our institution. There were three cases of bacteremia in this study in which the patient also had a positive urine culture. It is possible that in these cases, the patient's true source of infection may have been the urine and the positive blood culture may have been a contaminant. However, each of these cases met the study definition of bacteremia and was therefore, included in the analysis.

Our study showed only a statistically significant association between platelet count and bacteremia. One possible reason for this is that the study is powered to detect a $50-65 \%$ incidence of bacteremia, however, the actual incidence was higher. Therefore, more subjects may have been needed in order to detect a significant difference with regard to other variables.

Lastly, although difficult to determine its influence on bacteremia, TPN duration was not evaluated, nor was the length of remaining bowel determined for study subjects.

\section{Conclusion}

Pediatric GI patients with CVCs have a high incidence of CLABSIs in the setting of fever. The current study identified only platelet count as being associated with the presence of bacteremia. While the platelet count may be low in a patient in the setting of serious bacterial infection, there are other factors that may contribute to thrombocytopenia in the pediatric GI population. Therefore, thrombocytopenia alone cannot be used to reliably predict which patients may be bacteremic. While there were fewer patients in the current study requiring ICU admission than in previous studies, the high incidence of bacteremia is sufficient evidence to continue the current practice of admitting these patients on empiric antibiotics while awaiting culture and sensitivity results. A multi-center, prospective study would help to further understand this clinically important topic.

\section{Acknowledgements}

The authors would like to thank Dr. Todd Chang, MD for his contribution to this study.

\section{Funding}

This research did not receive any specific funding from agencies in the public, commercial, or not-forprofit sectors

\section{Authors Contributions}

CEB and ALN designed the study. CEB and JS performed the chart review and data extraction. CPW created the statistical design for this study and performed all data analysis. CEB, JS, and ALN all contributed to manuscript writing and editing. CPW also contributed to manuscript editing.

\section{References}

1. Alexander T, Blatt J, Skinner AC, Jhaveri R, Jobson M, et al. (2016) Outcome of pediatric gastroenterology outpatients with fever and central line. Pediatr Emerg Care 32: 746-750.

2. Eisenberg M, Monuteaux MC, Fell G, Goldberg V, Puder $M$, et al. (2018) Central line-associated bloodstream infection among children with intestinal failure presenting to the emergency department with fever. J Pediatr 196: 237-243. e1.

3. Szydlowski EG, Rudolph JA, Vitale MA, Zuckerbraun NS (2017) Bloodstream infections in patients with intestinal failure presenting to a pediatric emergency department with fever and a central line. Pediatr Emerg Care 33: e140-e145.

4. Moskalewicz RL, Isenalumhe LL, Luu C, Wee CP, Nager AL (2017) Bacteremia in nonneutropenic pediatric oncology patients with central venous catheters in the ED. Am J Emerg Med 35: 20-24.

5. Pironi L, Arends J, Baxter J, Bozzetti F, Peláez RB, et al. (2015) ESPEN endorsed recommendations. Definition and classification of intestinal failure in adults. Clin Nutr 34: 171180.

6. Squires RH, Duggan C, Teitelbaum DH, Wales PW, Balint $J$, et al. (2012) Natural history of pediatric intestinal failure: Initial report from the Pediatric Intestinal Failure Consortium. J Pediatr 161: 723-8.e2.

7. Wales PW, de Silva N, Kim J, Lecce L, To T, et al. (2004) Neonatal short bowel syndrome: Population-based estimates of incidence and mortality rates. J Pediatr Surg 39: 690-695.

8. Wilmore DW (1972) Factors correlating with a successful outcome following extensive intestinal resection in newborn infants. J Pediatr 80: 88-95.

9. Mohammed A, Grant FK, Zhao VM, Shane AL, Ziegler TR, et al. (2011) Characterization of posthospital bloodstream infections in children requiring home parenteral nutrition. JPEN J Parenter Enteral Nutr 35: 581-587.

10. Schalamon J, Mayr JM, Höllwarth ME (2003) Mortality and economics in short bowel syndrome. Best Pract Res Clin Gastroenterol 17: 931-942.

11. Miko BA, Kamath SS, Cohen BA, Jeon $\mathrm{C}$, Jia $\mathrm{H}$, et al. (2015) Epidemiologic associations between short-bowel syndrome and bloodstream infection among hospitalized children. J Pediatric Infect Dis Soc 4: 192-197.

12. Cole CR, Hansen NI, Higgins RD, Ziegler TR, Stoll BJ, et al. (2008) Very low birth weight preterm infants with surgical short bowel syndrome: Incidence, morbidity and mortality, 
and growth outcomes at 18 to 22 months. Pediatrics 122 : e573-e582.

13. Terra RM, Plopper C, Waitzberg DL, Cukier C, Santoro S, et al. (2000) Remaining small bowel length: Association with catheter sepsis in patients receiving home total parenteral nutrition: Evidence of bacterial translocation. World $\mathrm{J}$ Surg 24: 1537-1541.

14. Hise ME, Compher C, Harlan L, Kohlmeier JE, Benedict $\mathrm{SH}$, et al. (2006) Inflammatory mediators and immune function are altered in home parenteral nutrition patients. Nutrition 22: 97-103.

15. D'Antiga L, Dhawan A, Davenport M, Mieli-Vergani G, Bjarnason I (1999) Intestinal absorption and permeability in paediatric short-bowel syndrome: A pilot study. J Pediatr Gastroenterol Nutr 29: 588-593.

16. Wang P, Wang Y, Lu L, Yan W, Tao Y, et al. (2017) Alterations in intestinal microbiota relate to intestinal failure-associated liver disease and central line infections. J Pediatr Surg 52: 1318-1326.

17. Harris PA, Taylor R, Thielke R, Payne J, Gonzalez N, et al. (2009) Research electronic data capture (REDCap)--a metadata-driven methodology and workflow process for providing translational research informatics support. J Biomed Inform 42: 377-381.

18. Chang MI, Carlson SJ, Nandivada P, O'Loughlin AA, Potemkin AA, et al. (2016) Challenging the 48-Hour rule-out for central line-associated bloodstream infections in the pediatric intestinal failure population: $A$ retrospective pilot study. JPEN J Parenter Enteral Nutr 40: 567-573.

19. Robinson JL, Casey LM, Huynh HQ, Spady DW (2014) Prospective cohort study of the outcome of and risk factors for intravascular catheter-related bloodstream infections in children with intestinal failure. JPEN J Parenter Enteral Nutr 38: $625-630$

20. Bedet A, Razazi K, Boissier F, Surenaud M, Hue S, et al. (2018) Mechanisms of thrombocytopenia during septic shock: A multiplex cluster analysis of endogenous sepsis mediators. Shock 49: 641-648.

21. Mitchell O, Feldman DM, Diakow M, Sigal SH (2016) The pathophysiology of thrombocytopenia in chronic liver disease. Hepat Med 8: 39-50. 